Abanico Veterinario. Enero-Diciembre 2020; 10:1-11. http://dx.doi.org/10.21929/abavet2020.22 Artículo Original. Recibido: 20/01/2020. Aceptado: 19/08/2020. Publicado: 26/10/2020. Clave: 2020-6.

\title{
Frecuencia de enfermedades de impacto reproductivo en bovinos de doble propósito ubicados en Oaxaca, México
}

Frequency of reproductive impact diseases in dual-purpose cattle located in Oaxaca, Mexico

\section{Gutiérrez-Hernández José ${ }^{1}$ ID , Palomares-Resendiz Gabriela*1ID, Hernández- Badillo Erik $^{2 \text { ID }}$, Leyva-Corona José ${ }^{3}$ ID , Díaz-Aparicio Efrén ${ }^{1 \text { ID }}$, Herrera-López Enrique $^{1 \text { ID }}$}

${ }^{1}$ CENID Salud Animal e Inocuidad, Instituto Nacional de Investigaciones Forestales, Agrícolas y Pecuarias, Carretera Federal México-Toluca Km. 15.5, Cuajimalpa, Ciudad de México, 05110, México. 2 Facultad de Medicina Veterinaria y Zootecnia, Universidad Nacional Autónoma de México, Circuito Exterior de Ciudad Universitaria, Delegación Coyoacán, Ciudad de México, 04510, México. ${ }^{3}$ Departamento de Ciencias Agronómicas y Veterinarias del Instituto Tecnológico de Sonora. 5 de febrero 818 sur. CP 8500 . Ciudad Obregón, Sonora. *Autor responsable y correspondencia: Gabriela Palomares-Resendiz CENID Salud Animal e Inocuidad, Instituto Nacional de Investigaciones Forestales, Agrícolas y Pecuarias, Carretera Federal México-Toluca Km. 15.5, Cuajimalpa, Ciudad de México, 05110, México. palomares.erika@inifap.gob.mx, herrera.enrique@inifap.gob.mx, gutierrez.joseluis@inifap.gob.mx, gigiovanni10@hotmail.com,_jose.leyva@itson.edu.mx, diaz.efren@inifap.gob.mx

\section{RESUMEN}

La brucelosis, leptospirosis, diarrea viral bovina (DVB) y rinotraqueítis infecciosa bovina (IBR), son enfermedades abortivas que comprometen la eficiencia productiva en hatos bovinos. En diferentes municipios de Oaxaca, México, se han observado problemas reproductivos sugestivos a estas enfermedades. El objetivo del estudio fue determinar la frecuencia de DVB, IBR, brucelosis y leptospirosis en bovinos de doble propósito criados en diferentes regiones de Oaxaca. Se colectaron 2,691 muestras sanguíneas a partir de 127 hatos bovinos para diagnosticar serológicamente brucelosis mediante las pruebas de tarjeta al $8 \%$ y Rivanol; aglutinación microscópica (MAT) con una batería de seis serovariedades de Leptospira; ELISA por bloqueo e indirecta para DVB e IBR respectivamente. La frecuencia general aparente de hato fue brucelosis: $2.3 \%$, leptospirosis: $86.6 \%$, IBR: $65.4 \%$ y DVB: $56.7 \%$. La frecuencia real para brucelosis, leptospirosis, IBR y DVB fue de $-9.1 \%$ (IC $95=-10.1,-7.9 \%$ ), $64.3 \%\left(\mathrm{IC}_{95}=62.5,66.1 \%\right), 40.1 \%\left(\mathrm{IC}_{95}=38.2,41.9 \%\right)$ y $33.2 \%$ ( $\left.\mathrm{IC}_{95}=31.4,35.0 \%\right)$ respectivamente. En la región Costa se observó el mayor porcentaje de animales con anticuerpos contra Leptospira, IBR y DVB. El presente estudio evidenció serológicamente la presencia de anticuerpos contra brucelosis, IBR, DVB y seis serovariedades de Leptospira en hatos bovinos doble propósito del estado de Oaxaca, México.

Palabras clave: Brucelosis, leptospirosis, DVB, IBR, Frecuencia.

\begin{abstract}
Brucellosis, leptospirosis, bovine viral diarrhea (BVD) and bovine infectious rhinotracheitis (IBR) are abortive diseases that compromise productive efficiency in cattle. In different municipalities of Oaxaca, Mexico, reproductive problems suggestive of these diseases have been observed. This study determined the frequency of DVB, IBR, brucellosis and leptospirosis in dual-purpose cattle herds in different regions of Oaxaca Mexico. A total of 2,691 blood samples were collected from 127 bovine herds to diagnose brucellosis serologically using the rose bengal and Rivanol tests; microscopic agglutination test (MAT) with a battery of six Leptospira serovars; ELISA by blocking and indirect for BVD and IBR respectively. The apparent overall frequency of herd was brucellosis: $2.3 \%$, leptospirosis:
\end{abstract}


86.6\%, IBR: $65.4 \%$ and BVD: $56.7 \%$. The real frequency for brucellosis, leptospirosis, IBR and BVD was $-9.1 \%(\mathrm{Cl} 95=-10.1,-7.9 \%), 64.3 \%(\mathrm{Cl} 95=62.5,66.1 \%), 40.1 \%(\mathrm{IC} 95=38.2,41.9 \%)$ and $33.2 \%$ (IC95 $=31.4,35.0 \%)$ respectively. In the Costa region, the highest percentage of animals with antibodies against Leptospira, IBR and BVD was observed. The present study showed serologically the presence of antibodies against brucellosis, IBR, DVB and six serovars of Leptospira in bovine herds double purpose of the state of Oaxaca, Mexico.

Keywords: brucellosis, leptospirosis, BVD, IBR, frequency

\section{INTRODUCCIÓN}

Oaxaca ocupa el sexto lugar en el censo nacional ganadero con 1,741,741 bovinos, aunque la producción de carne y leche de esta especie se encuentra en la posición 12 y el 17 respectivamente (SIAP, 2018). Este estado se caracteriza por un clima tropical húmedo, donde predominan hatos doble propósito, tipo lechería familiar de 30 animales; pastoreados en especies nativas de temporal. En esta región de México, existen enfermedades de impacto reproductivo que ponen en riesgo la producción de becerros. Estos problemas sanitarios incrementan el costo de producción por concepto de tratamientos, baja tasa de parición y menor volumen de leche por pérdidas de gestación.

En México, más del $70 \%$ de los abortos se considerados de origen desconocido, aunado a estos problemas se suman los de salud, que comprometen la productividad eficiente de los animales (Escamilla et al., 2007). Dentro de las enfermedades más importantes están aquellas que afectan la reproducción, poniendo en riesgo la disponibilidad de becerros; además aumentan el costo de la producción por concepto de tratamientos (Rojo et al., 2009). Los agentes infecciosos asociados con desórdenes reproductivos en rumiantes, incluyen agentes virales abortivos, como Diarrea Viral Bovina (DVB) (Brodersen, 2014; Larghi, 2018) y Rinotraqueitis Infecciosa Bovina (IBR) (Baillargeon et al., 2017; Valas et al., 2019) de origen bacteriano a Brucella abortus (Zakia et al., 2016; Poester et al., 2013) y Leptospira (Martins y Lilenbaum., 2017; Lilenbaum y Martins., 2014). Por lo tanto, el objetivo del estudio fue determinar la frecuencia de anticuerpos contra brucelosis, leptospirosis, DVB e IBR en hatos bovinos doble propósito criados en diferentes regiones del estado de Oaxaca, México.

\section{MATERIAL Y MÉTODOS}

\section{Ubicación geográfica y animales de estudio}

El estudio se realizó en catorce municipios ubicados en la Sierra Norte, Itsmo y Costa, ubicados en el estado de Oaxaca (Cuadro 1). Se utilizaron 2,691 hembras bovinas de diferentes razas entre vaquillas con edad reproductiva (1.5 - 3 años) y adultas (De 3 a 12 años). Estos animales representan 217 hatos bovinos de doble propósito, tipo lechería familiar. Los hatos muestreados no tenían antecedentes de vacunación contra brucelosis (BRU), diarrea viral bovina (DVB), rinotraqueitis infecciosa bovina (IBR) y leptospirosis (LEP). 


\section{Diseño de estudio y muestreo}

El diseño del estudio fue observacional descriptivo, tipo transversal. Se realizó un muestreo no probabilístico (por conveniencia), en hatos de productores cooperantes. En cada bovino muestreado se colectaron $10 \mathrm{ml}$ de sangre (tubo desechable sin anticuagulante), mediante venopunción de la vena coccígea; utilizando equipo de extracción Vacutainer®. Las muestras fueron identificadas y colocadas en reposo durante 20 min aproximadamente a temperatura ambiente para el desprendimiento del coágulo, y después conservadas a $5^{\circ} \mathrm{C}$ para su transporte al laboratorio de enfermedades de los pequeños rumiantes, INIFAP, Palo Alto. Las muestras de sangre fueron centrifugadas a $20 \times \mathrm{g}$ por 10 min para la obtención de suero y almacenadas a $-5^{\circ} \mathrm{C}$ hasta los análisis serológicos.

\section{Pruebas serológicas}

Para el diagnóstico de brucelosis, se utilizó la prueba de tarjeta al 8\% (Aba test, PRONABIVE, México); las muestras positivas fueron confirmadas con la prueba de rivanol (Aba test PRONABIVE, México), considerando una muestra positiva, cuando los títulos eran $\geq 1: 50$ (NORMA Oficial Mexicana NOM-041-ZOO-1995).

El diagnóstico de leptospirosis se llevó a cabo empleando la prueba de aglutinación microscópica (MAT), utilizando seis serovariedades y tres cepas de referencia: Sejroe (serovariedad Wolffi), Sejroe (serovariedad Hardjo) y Tarassovi (serovariedad Tarassovi); así como tres de aislamiento nacional: Icterohaemorrhagiae (serovariedad Icterohaemorrhagiae), Sejroe (Hardjo prajitno) y Canicola (serovariedad Portlandvere); considerando una muestra positiva cuando los títulos eran $\geq 1: 100$ (OIE, 2004).

Para la detección y cuantificación de anticuerpos contra DVB, se utilizó una prueba comercial: CIVTEST® BOVIS BVD/BD P80 de Laboratorios Hipra, S.A., siguiendo las instrucciones del fabricante, incluidos los procedimientos para la determinación del índice relativo (determinado a partir de la densidad óptica (OD) de las muestras y controles. Es un ELISA de bloqueo que detecta anticuerpos frente a una proteína específica (p80) presente en todas las cepas del vDBV.

Las placas se leyeron con un lector de ELISA, con una longitud de onda de $450 \mathrm{~nm}$. Los resultados se expresaron en porcentaje de inhibición, de acuerdo a la siguiente fórmula: \% IN= (media DO de control negativo-DO muestra/media DO de control negativo) ${ }^{*} 100$.

Un porcentaje de inhibición menor a $50 \%$ es seronegativo.

Un porcentaje de inhibición igual o superior a $50 \%$ es seropositivo.

Para el diagnóstico de IBR se usó una prueba comercial, CIVTEST® BOVIS IBR de Laboratorios Hipra, S.A., siguiendo las instrucciones del fabricante, incluidos los procedimientos para la determinación del índice relativo (determinado a partir de la densidad óptica (OD) de las muestras y controles. La prueba se basa en un ELISA indirecto, que detecta anticuerpos específicos contra el virus de la IBR.

Las placas se leyeron con un lector de ELISA, con una longitud de onda de $450 \mathrm{~nm}$. Los resultados se expresan en índice relativo x 100 de acuerdo a la siguiente fórmula: 
IRPC $=(\mathrm{DO}$ muestra- media DO control negativo/media DO control positivo-media DO control negativo) ${ }^{*} 100$

Un Índice relativo por cien (IRPC) menor o igual a 9 es un resultado negativo. Un IRPC mayor de 9.0 e inferior a 15.0 es un resultado sospechoso.

Un IRPC mayor a 15 es un resultado positivo.

\section{Análisis estadístico}

Se construyeron tablas de contingencia para calcular la frecuencia de animales seropositivos por enfermedad y región. A partir del número de muestras positivas a cada una de las pruebas serológicas se estimó la frecuencia aparente a nivel hato $\left(p_{A H}\right)$ e individual aparente $\left(p_{I A}\right)$. Con el valor de la sensibilidad y especificidad de cada prueba utilizada, el número de animales seropositivos dentro de cada enfermedad, el tamaño de población de bovinos en el estado de Oaxaca $(\mathrm{N})$, tamaño de muestra $(\mathrm{n})$ por región y total de animales muestreados, se calculó la prevalencia real ( $\left.p_{\mathrm{re}}\right)$ y respectivos intervalos de confianza (IC95\%), para cada enfermedad dentro de cada región (Noordhuizen et al., 1997). Para el cálculo de los datos se procesaron en el programa WinEpi (Working in Epidemiology, http://www.winepi.net/ ) de acuerdo a la siguiente fórmula: $P_{r e}=$ Prevalencia Ind Aparente - (1- Esp)/1- [(1-Esp)+(1- Sens)]

Debido a que el muestreo no fue proporcional al tamaño del hato, la prevalencia $(p)$ y respectivo error estándar (E.E.p) fue corregido para el tamaño de hato mediante esta fórmula: $p=\sum N i p i / N$ y E.E. $p=\sqrt{D} * E . E . s$, donde $N i$ es el tamaño del hato; $p i$ la prevalencia del hato, $N=\sum N i$ es el número total de bovinos en los hatos muestreados; $D=$ el efecto del diseño $(D=1.96)$ (Bennett et al., 1991; Otte y Gumm, 1997); E.E.s el error estándar para un muestreo simple aleatorio $(\sqrt{ } p q / n) ; n$ es el número total de animales muestreados $(\mathrm{n}=2691)$. $D$ fue calculado como $D=1+(k-$ 1) $r_{e}$; siendo $k$ el número de animales del hato y $r_{e}$ la correlación dentro del hato, estimada a partir los componentes de un análisis de varianza de una vía que incluyó el efecto aleatorio del hato. El error estándar aproximado de re y $D$ se obtuvo de acuerdo a (Solis et al., 2003).

\section{Brucelosis}

\section{RESULTADOS}

En el cuadro 1, se presenta la frecuencia de brucelosis. Se observó una $p_{A H}=2.3 \%$ hatos positivos a brucelosis con la prueba de rivanol, lo que corresponde a una $p_{r e}=-$ $9.1 \%$ (IC95-10.1, -7.9\%). Lo anterior equivale a un total de cuatro animales positivos, siendo la región Costa donde se observó mayor frecuencia de animales y la Sierra Norte donde no hubo reactores.

\section{Leptospirosis}

El $86.6 \%$ de los hatos muestreados tuvo al menos un animal positivo a leptospirosis, para alguna de las seis serovariedades que fueron incluidas en este estudio (cuadro 1), equivalente a una $p_{r e}=64.3 \%$ (IC95 62.5, 66.1\%) en los animales muestreados. Las regiones Itsmo y Costa, tuvieron una $p_{A H}$ del $100 \%$; sin embargo, la región Sierra Norte fue la $p_{r e}$ más alta $(76.5 \%)$. Las serovariedades que tuvieron mayor frecuencia fueron 
Hardjo prajitno (49.09\%) e Icterohaemorrhagiae (34.89\%); ambas de aislamiento nacional (cuadro 2).

Cuadro 1. Frecuencia de enfermedades a nivel hato e individual calculada en cuatro regiones del estado de Oaxaca

\begin{tabular}{|c|c|c|c|c|c|c|c|c|c|}
\hline & & \multicolumn{3}{|c|}{ HATOS } & \multicolumn{5}{|c|}{ INDIVIDUAL } \\
\hline Enfermedad & Región & $\mathrm{n}$ & $(+)$ & $p_{A H}$ & $\mathrm{n}$ & $(+)$ & $p_{I A}$ & $p_{r e}$ & IC95\% \\
\hline \multirow{4}{*}{$\begin{array}{l}\text { Brucelosis } \\
\text { (Rivanol) }\end{array}$} & Sierra Norte & 120 & 0 & $0.0 \%$ & 1031 & 0 & $0.0 \%$ & -0.09 & $(-10.9,-7.4 \%)$ \\
\hline & Itsmo & 57 & 2 & $3.5 \%$ & 964 & 1 & $0.10 \%$ & $-9.07 \%$ & $(-10.9,-7.3 \%)$ \\
\hline & Costa & 40 & 3 & $7.5 \%$ & 696 & 3 & $0.43 \%$ & $-8.8 \%$ & $(-10.9,-6.72 \%)$ \\
\hline & Oaxaca & 217 & 5 & $2.3 \%$ & 2691 & 4 & $0.15 \%$ & $-9.1 \%$ & $(-10.1,-7.9 \%)$ \\
\hline \multirow{4}{*}{ Leptospirosis } & Sierra Norte & 120 & 91 & $75.8 \%$ & 1031 & 783 & $75.9 \%$ & $76.5 \%$ & $(73.9,79.1 \%)$ \\
\hline & Itsmo & 57 & 57 & $100 \%$ & 964 & 628 & $65.1 \%$ & $65.1 \%$ & $(62.0,68.1 \%)$ \\
\hline & Costa & 40 & 40 & $100 \%$ & 696 & 323 & $46.4 \%$ & $45.3 \%$ & $(41.5,48.9 \%)$ \\
\hline & Oaxaca & 217 & 188 & $86.6 \%$ & 2691 & 1734 & $64.4 \%$ & $64.3 \%$ & $(62.5,66.1 \%)$ \\
\hline \multirow{4}{*}{ IBR } & Sierra Norte & 120 & 50 & $41.7 \%$ & 1031 & 420 & $40.7 \%$ & $46.4 \%$ & $(43.4,49.5 \%)$ \\
\hline & Itsmo & 57 & 54 & $94.7 \%$ & 964 & 262 & $27.2 \%$ & $30.6 \%$ & $(27.7,33.5 \%)$ \\
\hline & Costa & 40 & 38 & $95.0 \%$ & 696 & 268 & $38.5 \%$ & $43.8 \%$ & $(40.1,47.5 \%)$ \\
\hline & Oaxaca & 217 & 142 & $65.4 \%$ & 2691 & 950 & $35.3 \%$ & $40.1 \%$ & $(38.2,41.9 \%)$ \\
\hline \multirow{4}{*}{ DVB } & Sierra Norte & 120 & 48 & $40.0 \%$ & 1031 & 430 & $41.7 \%$ & $40.8 \%$ & $(37.8,43.8 \%)$ \\
\hline & Itsmo & 57 & 38 & $66.7 \%$ & 964 & 307 & $31.8 \%$ & $30.3 \%$ & $(27.4,33.2 \%)$ \\
\hline & Costa & 40 & 37 & $92.5 \%$ & 696 & 194 & $27.9 \%$ & $26.0 \%$ & $(22.7,29.3 \%)$ \\
\hline & Oaxaca & 217 & 123 & $56.7 \%$ & 2691 & 931 & $34.6 \%$ & $33.2 \%$ & $(31.4,35.0 \%)$ \\
\hline
\end{tabular}

Para el cálculo de la Pre (prevalencia real) se consideró una $\mathrm{N}=1,766,208$ animales para el estado de Oaxaca (SIAP, 2018), una sensibilidad y especificidad por prueba de $83 \%$ y $93 \%$ para brucelosis (Rivanol); $98.2 \%$ y $96.4 \%$ para leptospirosis (MAT); $86.59 \%$ y $99.10 \%$ para IBR (ELISA); $96.94 \%$ y $97.84 \%$ para DVB (ELISA)

Cuadro 2. Frecuencia de animales seropositivos a cada una de las serovariedades de Leptospira identificadas por región

\begin{tabular}{lcccc}
\hline \multirow{2}{*}{ Serovariedad } & \multicolumn{3}{c}{ REGIÓN } & Total \\
\cline { 2 - 5 } & Sierra Norte & Costa & Istmo & $12.00 \%(316 / 2691)$ \\
\hline Canicola* & $2.03 \%(21 / 1031)$ & $32.76 \%(228 / 696)$ & $6.95 \%(67 / 964)$ & $27.60 \%(742 / 2691)$ \\
Hardjo & $25.80 \%(266 / 1031)$ & $19.68 \%(137 / 696)$ & $35.17 \%(339 / 964)$ & $49.00 \%(1321 / 2691)$ \\
Hardjo prajitno* & $53.34 \%(550 / 1031)$ & $37.64 \%(262 / 696)$ & $52.80 \%(509 / 964)$ & $34.90 \%(939 / 2691)$ \\
Icterohaemorrhagiae* & $57.13 \%(589 / 1031)$ & $35.78 \%(249 / 696)$ & $10.48 \%(101 / 964)$ & $5.80 \%(155 / 2691)$ \\
Tarassovi & $4.07 \%(42 / 1031)$ & $6.18 \%(43 / 696)$ & $7.26 \%(70 / 964)$ & $16.00 \%(442 / 2691)$ \\
Wolffi & $33.66 \%(347 / 1031)$ & $3.16 \%(22 / 696)$ & $7.57 \%(73 / 964)$ &
\end{tabular}

\section{Rinotraqueitis infecciosa bovina}

Se observó una $p_{A H}=65.4 \%$ en los hatos muestreados en el estado de Oaxaca, y una $p_{r e}=40.1 \%$ (IC $\left.9538.2,41.9 \%\right)$ de animales con anticuerpos contra IBR; aunque la $p_{A H}$ fue mayor en la región Costa (95\%) y la prefue más alta en la Sierra Norte (46.4\%). 


\section{Diarrea viral bovina}

El 56.7\% de los hatos tuvo anticuerpos contra DVB, con una $p_{r e}=33.2 \%$ (IC95 31.4, $35.0 \%$ ) en los bovinos muestreados. Un mayor porcentaje de hatos afectados fueron ubicados en la Costa (92.5\%); sin embargo, fue la región de la Sierra Norte donde se detectó la mayor $p_{r e}(40.8 \%$; IC95 37.8, 43.8\%) de anticuerpos contra DVB.

\section{DISCUSIÓN}

A pesar de la importancia económica que representan los bovinos para el estado de Oaxaca y el impacto que tienen las enfermedades reproductivas, la frecuencia de estas enfermedades era desconocida. Sin embargo, se han realizado varios trabajos en otras regiones del país sobre la frecuencia de las cuatro enfermedades estudiadas (Rosete et al., 2018; Segura et al., 2010; Segura et al., 2003)

En este trabajo se encontró evidencia serológica, aunque con una frecuencia baja de la presencia de Brucella, en estas regiones de Oaxaca. Los resultados son similares a los que reporta el Servicio Nacional de Sanidad, Inocuidad y Calidad Agroalimentaria (SENASICA, 2014), de 0.09\% para brucelosis bovina en dicho estado. En contraste, la prevalencia que reportan algunos autores en hatos lecheros de tipo intensivo, ubicados en zonas endémicas de esta enfermedad, son mucho más altos, influenciadas por el hacinamiento, falta de áreas exclusivas de parto, entre otras que favorecen la transmisión de la bacteria (Milián et al., 2016).

Los resultados de este trabajo demuestran una frecuencia serológica elevada de leptospirosis, DVB e IBR; que puede ser debido a la compra e introducción de bovinos provenientes de hatos infectados, la falta de barreras físicas, el contacto de los bovinos con otras producciones o no realizar cuarentena ni vacunación para evitar la aparición de manifestaciones clínicas de la enfermedad (Miyama et al., 2017; Milián et al., 2016; Gates et al., 2013; Lilenbaum y Martins., 2014; Muylkens et al., 2007; Nandi et al., 2009). Además, las condiciones de humedad que prevalecen en estas zonas favorecen la supervivencia de Leptospira fuera de los huéspedes, ocasionando que otros animales adquieran la infección. Con las pruebas serológicas no es posible determinar si la presencia de anticuerpos se debe a una infección reciente o de hace tiempo; lo que se puede concluir es que la gran mayoría de los animales están expuestos o viven en condiciones que permiten las infecciones con estos agentes, ya que en la mayoría de los hatos hubo por lo menos un animal seropositivo.

En los estudios que se realizan en bovinos es la serovariedad Hardjo, la que mayor frecuencia tiene, debido a que los bovinos son reservorios de esta serovariedad y la transmisión entre ellos se facilita por contacto directo y no depende de factores medioambientales (Carmona et al., 2011; Olmo, 2019). Segura et al., 2003 reportan una seroprevalencia de $62.8 \%$ en Yucatán; las serovariedades Hardjo y Tarassovi tuvieron la mayor seroprevalencia $54.1 \%$ y $53.3 \%$ respectivamente. Los resultados obtenidos en estos estudios coinciden con los obtenidos en el presente, siendo las serovariedades Hardjo, Wolffi e Icterohaemorrhagiae las diagnosticadas con mayor frecuencia (Carmona et al., 2011; Escamilla et al., 2007).

Los resultados de IBR son similares a los reportados, en estados de la zona sur del 
país; Solís et al., 2003 obtuvieron una seroprevalencia del 54.4\% en el estado de Yucatán; Milián et al., 2016 reportan una seroprevalencia entre el 57-83\% en ganado lechero en México. En estudios realizados al centro del país, las prevalencias reportadas difieren de nuestros resultados. Ojeda et al., 2016 en un estudio que realizaron para estimar la prevalencia de IBR en diferentes municipios del estado de México, reportaron 18\% de animales seropositivos, lo cual coincide con Magaña et al., 2005 quienes reportan una tasa del 22\% en bovinos de traspatio en Michoacán.

La frecuencia de la enfermedad varía en cada región, por lo que no se puede atribuir que el contacto estrecho sea el único factor que determine la alta prevalencia de la enfermedad, ya que se han reportado altas prevalencias de DVB e IBR en hatos lecheros y de doble propósito, donde los animales están en pastoreo (Milián et al., 2016).

La frecuencia de DVB en el país también presenta resultados variables. En este estudio se obtuvo una frecuencia de 35.3\%, Moles et al., 2002 reportaron $72.3 \%$ para DVB en bovinos de la zona centro de México, encontrando también presencia de anticuerpos contra IBR y leptospirosis; Romero et al., 2013 con 76.5\% en Veracruz; Segura et al., 2016 con 47.8\% en Tamaulipas; Escamilla et al., 2007 con $70 \%$ en Querétaro; Meléndez et al., 2010 con 32.8\% en Aguascalientes; Segura et al., 2010 con $16.4 \%$ en Michoacán, Milián et al., 2016 reporta una prevalencia del $79 \%$ en diferentes sistemas de producción en varios estados de la república mexicana. Rosete et al., 2018 en un estudio realizado en Veracruz, Puebla y Tabasco; reportan que el $100 \%$ de los hatos presentaron anticuerpos contra DVB, por lo que sugieren que el virus de la DVB está ampliamente distribuido en los tres estados.

En base a los resultados de este estudio se evidenció serológicamente la presencia de anticuerpos contra IBR, DVB y seis serovariedades de Leptospira en la población de estudio. En la región Sierra Norte se detectó el mayor porcentaje de animales con anticuerpos contra leptospirosis, diarrea viral bovina y rinotraqueitis infecciosa bovina; pero fue la región de la Costa donde se detectó mayor distribución de las cuatro enfermedades del estudio. Se debe realizar el aislamiento e identificación de los agentes causantes para que se implementen estrategias de prevención y control recomendables en cada caso.

\section{CONCLUSIONES}

El estudio determinó la presencia de anticuerpos contra leptospirosis (86.6\%), IBR (65.4\%), DVB (56.7\%) y brucelosis $(2.3 \%)$ en hatos bovinos de doble propósito, ubicados en diferentes municipios del Estado de Oaxaca, México. El nivel de distribución, frecuencia e impacto reproductivo y económico de estas enfermedades sugiere la implementación de medidas sanitarias que permitan prevenirlas y controlarlas.

\section{AGRADECIMIENTOS}

Este proyecto se realizado gracias al financiamiento obtenido de: Fundación Produce Oaxaca. FOP/GG/896/2012. Validación de la vacuna contra la brucelosis en bovinos de carne y doble propósito para determinar la causa de abortos en regiones ganaderas de Oaxaca, México. 


\section{LITERATURA CITADA}

BENNETT S, Woods T. Liyanage WM, Smith DL. 1991. A simplified general method for cluster-sampling surveys of health in developing countries. World Health Stat. Quart. 44(3):98-106. https://pubmed.ncbi.nlm.nih.gov/1949887/

BAILLARGEON P, Arango-Sabogal, JC, Wellemans V, Fecteau G. 2017. Determining bovine viral diarrhea and infectious bovine rhinotracheitis infections in dairy cattle using precolostral blood. The Canadian Veterinary Journal. 58(4): 360-364. https://pubmed.ncbi.nlm.nih.gov/28373727/

BRODERSEN WB. 2014. Bovine Viral Diarrhea Virus Infections: Manifestation of Infection and recent advances in understanding pathogenesis and control. Veterinary Pathology. 51(2):453-464. https://doi.org/10.1177/0300985813520250

CARMONA GC, León LL, Castillo SL, Ramírez OJ, Ko A, Lua PC, De la Peña MA. 2011. Detection of Leptospira santarosai y L. kirshneri in cattle: new isolates with potential impact in bovine production and public health. Veterinaria México. 42(4): 277288. http://www.scielo.org.mx/pdf/vetmex/v42n4/v42n4a3.pdf

ESCAMILLA HP, Martínez MJ, Medina MC, Morales SE. 2007. Frequency and causes of infectious abortion in a dairy herd in Queretaro, Mexico. Canadian Journal of Veterinary Research. 71(4):314-317.

https://www.ncbi.nlm.nih.gov/pmc/articles/PMC1940280/pdf/cjvr71_pg314.pdf

GATES MC, Woolhouse ME, Gunn GJ, Humphry RW. 2013. Relative association of cattle movements, local spread, and biosecurity with bovine viral diarrhea virus (BVDV) seropositivity in beef and dairy herd. Preventive Veterinary Medicine. 112(3-4):285295. https://doi.org/10.1016/j.prevetmed.2013.07.017

LARGHI M. 2018. Comparative study in the control of bovine viral diarrhea. Animal Health Research Reviews. 19:125-133. https://doi.org/10.1017/S1466252318000129

LILENBAUM W, Martins G. 2014. Leptospirosis in Cattle: A challenging scenario for the understanding of the epidemiology. Transboundary and Emerging Diseases. 61 Suppl 1:63-8. https://doi.org/10.1111/tbed.12233

MAGAÑA UA, Solorio RJL, Segura CJC. 2005. Rinotraqueítis infecciosa bovina en hatos lecheros de la región Cutzio-Téjaro, Michoacán, México. Técnica Pecuaria México. 43(1):27-37. http://www.redalyc.org/articulo.oa?id=61343103

MARTINS G, Lilenbaum W. 2017.Control of bovine leptospirosis: Aspects for consideration in a tropical environment. Research in Veterinary Science. 112:156-160. https://doi.org/10.1016/j.rvsc. 2017.03.021 
MELÉNDEZ SR, Valdivia FA, Rangel ME, Díaz AE, Segura CJ, Guerrero BA. 2010. Factores de riesgo asociados a la presencia de aborto y desempeño reproductivo en ganado lechero de Aguascalientes, México. Técnica Pecuaria en México. 1(4):391401. http://www.scielo.org.mx/pdf/rmcp/v1n4/v1n4a7.pdf

MILIÁN SF, Hernández OR, Hernández AL, Alvarado IA, Díaz AE, Mejía EF, Palomares RE, Bárcenas RI, Zendejas MH. 2016. Seroprevalence and risk factors for reproductive diseases in dairy cattle in Mexico. Journal of Veterinary Medicine and Animal Health. 8(8):89-98. https://doi.org/10.5897/JVMAH2016.0483

MIYAMA T, Watanabe E, Ogata Y, Urushiyama Y, Kawahara N, Makita K. 2017. Herdlevel risk factors associated with Leptospira Hardjo infection in dairy herds in the southern Tohoku, Japan. Preventive Veterinary Medicine. 149:15-20. doi:10.1016/j.prevetmed.2017.11.008

MOLES CL, Gavaldón D, Torres BJ, Cisneros PM, Aguirre SJ, Rojas SN. 2002. Seroprevalencia simultánea de Leptospirosis y tres enfermedades de importancia reproductiva en bovinos del altiplano central de la República Mexicana. Universidad Autónoma Metropolitana Xochimilco. Revista de Salud Animal. 24(2):106-110. https://biblat.unam.mx/es/buscar/seroprevalencia-simultanea

MUYLKENS B, Thiry J, Kirten P, Schynts F, Thiry E. 2007. Bovine herpesvirus 1 infection and infectious bovine rhinotracheitis. Veterinary Research. 38(2):181-209. https://doi.org/10.1051/vetres:2006059

NANDI S, Kumar M, Manohar M, Chauhan R. 2009. Bovine herpesvirus infections in cattle. Animal Health Research Reviews. 10(1):85-98. https://doi.org/10.1017/S1466252309990028

NOORDHUIZEN JP, Frankena K, Hoofd CM, Graat EA. 1997. Aplication of quantitaive methods in veterinary epidemiology. Published by: Wageningen Pers. (Wagenigen). The Netherlands pp.445. ISBN 0974134351. https://library.wur.nl/WebQuery/wurpubs/109308

OIE. 2004. Manual de la OIE sobre animales terrestres. Leptospirosis. Organización Mundial de Sanidad Animal. https://www.oie.int/doc/ged/d6508.pdf

OJEDA CJ, Espinosa AE, Hernández GP, Rojas MC, Álvarez MJ. 2016. Seroprevalencia de enfermedades que afectan la reproducción de bovinos para leche con énfasis en neosporosis. Ecosistemas y recursos agropecuarios. 3(8):243-249. http://www.scielo.org.mx/pdf/era/v3n8/2007-901X-era-3-08-00243.pdf

Olmo L, Reichel MP, Nampanya S, Khounsy S, Wahl LC, Clark BA, et al. 2019. Risk factors for Neospora caninum, bovine viral diarrhoea virus, and Leptospira interrogans serovar Hardjo infection in smallholder cattle and buffalo in Lao PDR. PLoS ONE 14(8): e0220335. https://doi. org/10.1371/journal.pone.0220335 
OTTE MJ, Gumm ID. 1997. Intra-cluster correlation coefficients of 20 infections calculated from the results of cluster-sample surveys. Preventive Veterinary Medicine. 31:147-150. doi: 10.1016/s0167-5877(96)01108-7

POESTER FP, Samartino LE, Santos RL. 2013. Pathogenesis and pathobiology of brucelosis in livestock. Revue Scientifique et Technique Office International des Epizooties. 32(1):105-115. https://pdfs.semanticscholar.org/f889/696c62f6fa0dfa5ac501fd05250aea4fb041.pdf

ROMERO SD, Ahuja AC, Montiel PF, García VZ, Cruz RA; Aguilar DM. 2013. Seroprevalence and risk factors associated with infectious bovine rhinotracheitis in unvaccinated cattle in southern Veracruz, Mexico. African Journal of Microbiology Research. 7(17):1716-1722.

http://www.academicjournals.org/app/webroot/article/article1380540810_RomeroSalas\%20et\%20al.pdf

ROJO RR, Vázquez A JF, Pérez HP, Mendoza MGD, Salem MAZ, Albarrán PB, González RA, Hernández MJ, Rebollar RS, Cardoso JD, Dorantes CEJ, Gutiérrez CJG. 2009. Dual Purpose cattle production in Mexico. Tropical Animal Health and Production. 41:715-721. https://doi.org/10.1007/s11250-008-9249-8

ROSETE FJ, Utrerab RA, Martínez ZJ, Jenkinsa OS, Zuritac GL, Islasa FA, Banda RB, Socci EG. 2018. Prevalencia de anticuerpos contra diarrea viral bovina en vacas no vacunadas en los estados de Puebla, Tabasco y Veracruz, México. Revista Mexicana de Ciencias Pecuarias. 9(3). https://doi.org/10.22319/rmcp.v9i3.4599

SOLIS CJ, Segura CVM, Segura CJC, Alvarado IA. 2003. Seroprevalence of and risk factors for infectious bovine rhinotracheitis in beef cattle herds of Yucatan, Mexico.

Preventive Veterinary Medicine. Apr 15;57(4):199-208. https://doi.org/10.1016/s01675877(02)00230-1

SEGURA CV, Solís CJJ, Segura CJ. 2003. Seroprevalence of and risk factor for leptospiral antibodies among cattle in the state of Yucatan, Mexico. Tropical Animal Health and Production. 35, 293-299. https://doi.org/10.1023/a:1025185703587

SEGURA Correa JC, Solorio Rivera JL, Sánchez Gil LG. 2010. Seroconversion to bovine viral diarrhoea virus and infectious bovine rhinotracheitis virus in dairy herds of Michoacan, Mexico. Tropical Animal Health and Production. 42:233-238. https://doi.org/10.1007/s11250-009-9411-y

SEGURA CJ, Zapata CC, Jasso OJ, Martínez BJ; López ZR. 2016. Seroprevalence and risk factors associated with bovine herpesvirus 1 and bovine viral diarrhea virus in North-Eastern Mexico. Open Veterinary Journal. 6(2):143-149. http://doi.org/10.4314/ovj.v6i2.12 
SERVICIO Nacional de Sanidad, Inocuidad y Calidad Agroalimentaria. 2014. Dirección general de salud animal. Dirección de campañas zoosanitarias, datos de frecuencias. Servicio Nacional de Sanidad, Inocuidad y Calidad Agroalimentaria. SAGARPA. https://www.gob.mx/senasica/documentos/informes-zoosanitarios-semanales-2014

SIAP. Servicio de Información Agroalimentaria y Pesquera. 2018. Bovinos carne y leche, Población ganadera 2008-2017 cabezas. Servicio de Información Agroalimentaria y Pesquera. www.gob.mx/siap/documentos/poblacion-ganadera136762

SOLíS CJ, Segura CV, Segura CJ, Alvarado IA. 2003. Seroprevalence of and risk factors for infectious bovine rhinotracheitis in beef cattle herds of Yucatan, Mexico. Preventive Veterinary Medicine. 57(4):199-208. https://doi.org/10.1016/S01675877(02)00230-1

VALAS S, Brémaud I, Stourm S, Croisé B, Mémeteau S, Ngwa-Mbot D, Tabouret M. 2019. Improvement of eradication program for infectious bovine rhinotracheitis in France inferred by serological monitoring of singleton reactors in certified BoHV1-free herds. Preventive Veterinary Medicine. 171:104743. https://doiorg.pbidi.unam.mx:2443/10.1016/j.prevetmed.2019.104743

ZAKIA I, Goodwin D, Pascual W. 2016. Brucellosis vaccines for livestock. Veterinary Immunology and Immunopathology. 181 (15): 51-58. https://doi.org/10.1016/j.vetimm.2016.03.011 